\title{
DOWNREGULATION OF TOLL-LIKE RECEPTOR 4 AND ACUTE PHASE CYTOKINES MEDIATED DEXAMETHASONE IMMUNOSUPPRESSANT MECHANISM DURING CRYPTOSPORIDIUM-PARVUM INFECTION
}

\author{
By \\ TAREK K. ZAALOUK ${ }^{1 *}$, GAMAL A. ABO SHEISHAA ${ }^{1}$, \\ AND IBRAHIM R. SHALASH ${ }^{2}$
}

Department of Medical Parasitology, Faculty of Medicine, Al-Azhar University ${ }^{1}$, Nasr City, Cairo, and Theodor Bilharz Research Institute ${ }^{2}$, Imbaba P.O.B. 30, Giza, Egypt

( ${ }^{*}$ Correspondence: tkzaalouk@gmail.com).

\section{Abstract}

TLRs in enterocytes play an important role in protecting the mucosal surface by promoting the innate inflammatory immune response following infection. Cryptosporidium parvum generally causes a short-term diarrheal illness that resolves spontaneously in immunocompetent hosts. But, in immunocompromised hosts, (e.g. on steroid therapy), it may cause a life-threatening, chronic, cholera-like illness. The mechanisms by which steroids reduced the host resistance to C. parvum infection are poorly understood. It was hypothesized that steroid may interfere with TLR activation and reduced proinflammatory cytokines production required for early infection containment. To test this hypothesis, sixty $\mathrm{C} 57 \mathrm{BL} / 6$ mice were equally divided into three subgroups; G1 was daily given intraperitoneally dexamethasone (DEX) for 14 days before oocyst inoculation and throughout the study. G2 was non-immunosuppressed mice. G1 \& G2 were inoculated intragastrically with C. parvum $\left(10^{6}\right.$ oocysts). G3 was retained as naïve non-infected control. Length and severity of infections were determined by monitoring oocyst shedding in fecal pellets using acid-fast (Ziehl-Neelsen) stained smear prepared from fresh pellets, by counting the number of oocysts in 10 microscopic fields under $\mathrm{x} 100$ for each mice group. Expression of TLR4 was assessed by immunohistochemistry, serum of proinflammatory cytokines level was determined by ELISA. The results showed that DEX immunosuppressed mice developed chronic cryptosporidiosis that lasted until the study end. Non-immunosuppressed mice developed a patent infection and the majority of them overcame the infection by the $21^{\text {st }}$ day. The mean oocyst count was significantly higher in the immunosuppressed mice than in non-immunosuppressed ones, with a longer patent period in immunosuppressed mice.

Expression of TLR4 was significantly increased in mice gut post-infection. Expression of TLR was significantly decreased in DEX immunosuppressed infected mice, with a significant decr-

ease was in proinflammatory cytokines serum levels compared to non-immunosuppressed ones.

Keywords: Mice, Cryptosporidium parvum, Toll-Like Receptor, Acute phase cytokines.

\section{Introduction}

Cryptosporidiosis is a ubiquitous zoonotic disease particularly common in young children in developing countries (Striepen, 2013) including Egypt (Abaza et al, 2016). Man acquired infection by oocysts of $C$. parvum or $C$. hominis that infects many mammalian hosts as an infectious disease (Chalmers and Davies, 2010). Infection is transmitted fecal-orally as well as from contaminated vegetables and water (El-Shazly et al, 2007). In the intestine, four sporozoites were excysted, infected epithelial cells and initiate asexual development. They become internalized and underwent merogony producing eight merozoites. Merozoites from this type I meront can either produce another type I or develops a type II meront with four merozoites. The four merozoites released from the type II merogony gave rise to sexual developmental stages, the micro- and macrogamonts. The release of microgametes, and their union with macrogametes, gives rise to the zygote, which, after two asexual divisions, forms the environmentally resistant oocyst containing four sporozoites while still within the host cell (O'Donoghue, 1995; Tzipori and Griffiths 1998). Newborn mice are highly susceptible to infection, but in adult wild type animals, $C$. parvum develops poorly (Heine et al, 1984). The parasite multiplies rapidly in the newborn mice 
for several days before the infection is brought under control. Innate immune response in neonatal mice is capable to cause C. parvum infection under highly effective control. But, $\mathrm{CD}^{+} \mathrm{T}$ cells were required for ultimate parasite elimination (McDonald et $a l, 2013)$. The initial host resistance to infection is to a large extent immunologically mediated as treatment with immunosuppressive drugs or certain cytokine-neutralizing antibodies rapidly exacerbates the infection (Ungar et al, 1991; McDonald et al, 1994). The Toll-like receptors (TLRs) family plays a key role in the innate immune system. These pattern recognition receptors recognize evolutionarily conserved pathogenassociated molecular patterns present on most types of microorganisms (Iwasaki and Medzhitov, 2004). Once TLRs are activated, they signal to the host the presence of infection and trigger signaling cascades leading to the induction of the appropriate acute phase cytokines of the early innate immune response (Akira, and Takeda, 2004). The TLR ligand engagement results in intracellular signal transduction, including activation of the nuclear factor B (NF-B) and mitogenactivated protein kinases (MAPKs). The TLR- activated signaling pathways proceed via adaptor proteins (most importantly MyD88) and lead to activation of the MAPKs and the inhibitory B (IB) protein kinase (IKK) complex. IKK, in turn, phosphorylates IB and targets it for degradation, hence liberating NF-B, which migrated to the nucleus and activated the transcription of target genes (Narayanan and Park, 2015). The intestinal epithelial cells were found to express TLRs and infection by $C$. parvum induced the recruitment of TLR2 and TLR4 to the site of infection (Sato et al, 2003). Knockdown of MyD88, TLR2 \& TLR4 expression resulted in inhibition of the downstream signaling pathways and thus increased the $C$. parvum infection (Chen et al, 2005). Dexamethasone (DEX) is widely used due to its pot- ent anti-inflammatory and immunosuppressive effects. Dexame- thasone immunosuppressed mice experience chronic cryptosporidial infection which eventually fatal. But, the mechanism of this effect was still not well known (McDonald et al, 2013)

The present study aimed to evaluate the dexamethasone interference with the host defense to C. parvum by downregulation of TLR4 on enterocytes and suppressing proinflammatory cytokines production in mice.

\section{Material and Methods}

Ethics statement: The study was conducted at Theodor Bilharz Research Institute (SBSP /TBRI, Giza, Egypt) in strict accordance with the TBRI Guidelines for Ethical Conduct in Use of Animals in Research.

Animals: Sixty specific pathogen-free C57 BL/6 mice aged 8 weeks and weighing 18$20 \mathrm{~g}$. were divided into equal three groups and maintained in conditioned rooms at $21^{\circ} \mathrm{C}$ on sterile water and a balanced dry food containing $14 \%$ protein. They were housed in groups of two to three mice each in wire- floored cages with underlying moist towels to collect fecal pellets (Petry et al, 1995). For immunosuppression, mice were given DEX intraperitoneally at a dose of $125 \mathrm{ug} /$ day for 14 days before oocyst inoculation. All mice continued to receive DEX after oocyst inoculation throughout the experimental time (Rasmussen and Healey, 1992).

Parasites and infection: Purified C. parvum oocysts in phosphate-buffered saline (PBS) pH7.2 were obtained from Theodor Bilharz Research Institute (SBSP/TBRI, Giza) and stored at $4^{\circ} \mathrm{C}$. Oocysts were surface sterilized by suspension in $10 \%$ (vol/ vol) commercial bleach solution (sodium hypochlorite), washed three times in PBS, and enumerated in the Neubauer hemocytometer. Mice were infected by oral gavage, using $1 \times 10^{6}$ C. parvum oocysts (Barakat et al, 2009).

Determination of oocyst shedding in infected mice: Fecal pellets were collected from mice to monitor oocyst shedding throughout the experiment. Pellets were re-suspended in 
a volume of $2.5 \%$ potassium dichromate approximately equal to twice that of the feces and stored at $4{ }^{\circ} \mathrm{C}$. Fecal suspensions were smeared onto slides and examined microscopically for the stained oocysts by acidfast (Ziehl-Neelsen) stain. The number of oocysts in 10 fields under the x100 oil immersion objective lens was calculated for each group of mice (Mc-Donald et al, 1994). Immunohistochemistry: The terminal $2 \mathrm{~cm}$ of the ileum was submitted to routine histopathological processing at the Department of Pathology, TBRI, where they were fixed in $10 \%$ neutral buffered formalin, dehydrated in ascending grades of ethanol, followed by immersion in xylene, and then impregnated in paraffin. Two 5-mm thick sections were taken, deparaffinized in xylene and rehydrated in ethanol followed by water and PBS. Endogenous peroxidase was blocked by immersion in 3\% hydrogen peroxide. Tissue sections were incubated with TLR4 antibody $(\mathrm{Ab})$ at a concentration of $5 \mu \mathrm{g} / \mathrm{ml}$ for $1 \mathrm{hr}$ at room temperature. Control sections were incubated with PBS with normal goat serum but without a primary $\mathrm{Ab}$. Immunostaining was detected according to Vectastain peroxidase kit (Vector Lab) and developed with diaminobenzidine tetrahydrochloride. Sections were counterstained with hematoxylin followed by light microscopy (Herman and Elfont, 1991).

Cytokines assay: Serum concentrations of proinflammatory cytokines IL-1 $\alpha$, IL6 \& TNF- $\alpha$ were determined by ELISA (Biosource International, Camarillo, California, USA) as recommended by the supplier.

Statistical analysis: Data were collected, tabulated, and statistically analyzed by using statistical package for the social sciences program, version 18 (SPSS; SPSS Inc. Chicago, Illinois, USA). Data were expressed as means and SD. Analysis was carried out using a two-tailed Student's $t$-test for unpaired means. Significant was considered when a $P$ value less than 0.05 .

\section{Results}

The C. parvum infection pattern in DEX immunosuppressed mice compared to the non-immunosuppressed ones. C57BL/6 mice proved a suitable animal model to study potential anti cryptosporidial agents, and elucidating the immunological defects that allowed $C$. parvum to be chronic infections (Petry et al, 1995). The study determined $C$. parvum infections pattern in DEX immunosuppressed C57BL/6 mice compared to nonimmunosuppressed mice. First group was given DEX intraperitoneally for 14 days at a dosage of $125 \mathrm{ug} /$ day before oocyst inoculation and continued to receive drug until the experimental end. Second group was nonimmunosuppressed mice, both groups were inoculated intragastrically with $10^{6}$ oocysts. Third group was non-infected and nontreated control mice. Each group consi-sted of 20 mice, and the experiment was repeated twice. Length and severity of infections were determined microscopically by monitoring oocyst shedding in fecal pellets. Acid-fast (Ziehl-Neelsen) stained fecal smear was prepared from fresh pellets. Oocysts were counted in 10 microscopic fields under x100 oil immersion. Shedding oocysts intensities were significantly greater in DEX immunosuppressed than in non-immunosuppressed mice. Both mice groups started oocyst shedding on the $4^{\text {th }}$-day p.i. (not shown). Non-immunosuppressed mice decreased shedding after day 7 post-infection, and majority recovered on the $21^{\text {st }}$ day, but immunosuppressed mice continued to shed oocysts throughout the experimental time (Fig. 1).

Murine TLR4 expression during C. parvum infection: Studies investigated TLR4 regulation in murine models of $C$. parvum infection. Infected DEX immunosuppressed, infected non-immunosuppressed and controls non-infected C57BL/6 mice were subjected to the experiments. Each group consisted of 20 mice, and the experiment was repeated twice. Constitutive mild expression of TLR4 occurred in gut of uninfected control mice (Fig. 2a). The expression of TLR4 was significantly increased in intestinal cells on day 7 post-infection (Fig 2b). In contrast, expre- 
ssion of TLR was significantly decreased in infected mice given dexamethasone treatment when compared to mice exposed to parasites only (Fig. 2c).

Dexamethasone down-regulated sera levels of acute-phase cytokines during infection. C. parvum induced a strong inflammatory response in intestine by TLR activation (Mc-Donald et al, 2013). The C. parvum infection increased serum levels of IL- $1 \alpha$, TNF- $\alpha$ \& IL-6 in nonimmunosuppressed mice. In contrast, the serum levels of cytokines were significantly decreased in the DEX immunosuppressed animals (Fig. 3a-c).

\section{Discussion}

No doubt, adaptive immunity is essential for complete recovery from $C$. parvum infection, innate immune responses play a crucial role in resistance against the parasite (Mogensen et al, 2008). One of the central components of innate immune system is the family of Toll-like receptors (TLRs). TLRs in intestinal epithelial cells protect the mucosal surface by acting as important inflammatory sensors of specific molecular structures of microbial pathogens and initiating innate inflammatory response (Santaolalla and Abreu, 2012). Steroids interfere with the activation of the TLR (Broering et al, 2011). The inhibition of TLRs signaling was the main target for anti-inflammatory and immunosuppressive effect of dexamethasone (Eicher et al, 2004; Broering et al, 2011).

The present study showed that DEX immunosuppressed mice developed a more severe clinical cryptosporidiosis course with infected longer and shed more oocysts than immunocompetent mice. This agreed with Adell et al. (2013) who found that DEX immunosuppressed mice developed chronic C. parvum infection persisted over 10 experimental weeks.

Regulation of TLR4 expression during $C$. parvum infection was investigated. Constitutive mild expression of TLR4 was observed in naïve non-infected mice. In non-immunosuppressed mice; C. parvum induced marked increase in intestinal expression of TLR4.
In DEX immunosuppressed mice, TLR4 expression was severely impair-ed. So, downregulation of TLR4 expression by DEX may be an immune defect that utilized by $C$. parvum to establish a chronic infection. C. parvum infection of cholangiocytes induced the selective recruitment of TLR2 \& TLR4 to infection sites, and suggested that TLRs mediate cholangiocyte defense responses to $C$. parvum via activation of NF-kappaB (Chen et al, 2005). As TLRs orchestrate the induction of appropriate acute phase cytokines of the early innate immune response, effects of DEX inhibition of TLR4 on production of proinflammatory cytokines (TNF- $\alpha$, IL-6, IL-1 $\alpha$ ) was analyzed. A marked decrease in serum levels of cytokines was seen in DEX immunosuppressed mice, that might be due to down-regulation of TLR 4 expression in mice. Br-oreing et al. (2011) found that steroids were potent modulators of TLR-mediated activation of liver cells resulting in suppression of pro-inflammatory and induction of anti-inflammatory cytokines.

\section{Conclusion}

The outcome data showed that steroids interfered with host resistance to C. parvum infection by suppression of TLR-induced production of pro-inflammatory cytokines.

\section{References}

Abaza, BE, Hamza, RS, Farag, TI, Abdel-Hamid, MA, Moustafa, RA, 2016: Assessing the efficacy of nitazoxanide in the treatment of cryptosporidiosis using PCR examination. J. Egypt. Soc. Parasitol. 46, 3:683-692.

Adell, AD, Miller, WA, Harvey, DJ, Vanwormer, E, Wuertz, S, et al, 2013: Individual subject meta-analysis of parameters of Cryptosporidium parvum shedding and diarrhea in animal experimental models. Epidemiol Infect. 141, 8: 1662-78.

Akira, S, Takeda, K, 2004: Toll-like receptor signaling. Nat. Rev. Immunol. 4:499-511.

Barakat, FM, McDonald, V, Di Santo, JP, Korbel, DS, 2009: Roles for NK cells \& an NK cell-independent source of intestinal gammainterferon for innate immunity to Cryptosporidium parvum infection. Infect Immun. 77: 5044-9.

Broering, R, Montag, M, Jiang, M, Lu, M, Sowa, JP, et al, 2011: Corticosteroids shift the 
Toll-like receptor response pattern of primaryisolated murine liver cells from an inflammatory to an anti-inflammatory state. Int. Immunol. 23, 9:537-44.

Chalmers, RM, Davies, AP, 2010: Mini-review: Clinical cryptosporidiosis. Exp. Parasitol. 124:138-46.

Chen, XM, Hara, SP, Nelson, JB, 2005: Multiple TLRs are expressed in human cholangiocytes and mediate host epithelial defense responses to Cryptosporidium parvum via activation of NF-kappaB. J. Immunol. 175:7447-56.

Eicher, SD, Mcmunn, KA, Hammon, HM, Donkin, SS, 2004: Toll-like receptors 2 and 4, and acute phase cytokine gene expression in dexamethasone and growth hormone-treated dairy calves. Vet. Immunol. Immunopathol. 98: 115. doi: 10.1016/j.vetimm.2003.10.009.

El Shazly, AM, Elsheikha, HM, Soltan, DM, Mohammad, KA, Morsy, TA, 2007: Protozoal pollution of surface water sources in Dakahlia Governorate, Egypt. J. Egypt. Soc. Parasitol. 37, 1:55-64.

Heine, J, Moon, HW, Woodmansee, DB, 1984: Persistent Cryptosporidium infection in congenitally athymic (nude) mice. Infect. Immun. 43:856-9.

Herman, GE, Elfont, EA, 1991: The taming of immunohistochemistry: The new era of quality control. Biotec. Histochem. 66:194-9.

Iwasaki, A, Medzhitov, R, 2004: Toll-like receptor control of the adaptive immune responses.

Nat. Immunol. 5:987-95.

Jiang, Z, Zamanian-Daryoush, H, Nie, AM, Silva, B, Williams, R, et al, 2003: Poly (I-C)induced Toll-like receptor 3 (TLR3)-mediated activation of NFB and MAP kinase is through an interleukin-1 receptor-associated kinase (IRAK)independent pathway employing the signaling components TLR3-TRAF6-TAK1-TAB2-PKR. J. Biol. Chem. 278:16713-9.

McDonald, V, Bancroft, GJ, 1994: Mechanisms of innate and acquired resistance to Cryptosporidium parvum infection in SCID mice. Parasite Immunol. 16:315-20.

McDonald, V, Korbel, DS, Barakat, FM, Choudhry, N, Petry, F, 2013: Innate immune res- ponses against Cryptosporidium parvum infection. Parasite Immunol. 35:55-64.

Mogensen, TH, Berg, RS, Paludan, SR, Østergaard, L, 2008: Mechanisms of dexamethasone-mediated inhibition of Toll-like receptor signaling induced by Neisseria meningitis and Streptococcus pneumoniae. Infect. Immun. 76, 1:189-97.

Narayanan, KB, Park, HH, 2015: Toll/ interleukin-1 receptor (TIR) domain-mediated cellular signaling pathways. Apoptosis. 20, 2:196-209 O'Neill, LA, 2006: How Toll-like receptors signal: What we know and what we don't know. Curr. Opin. Immunol. 18:3-9.

O'Donoghue, PJ, 1995: Cryptosporidium and cryptosporidiosis in man and animals. Int. J. Parasitol. 25, 2:139-45.

Petry, F, Robinson, HA, McDonald, V, 1995: Murine infection model for maintenance and amplification of Cryptosporidium parvum oocysts. J. Clin. Microbiol. 33:1922-4.

Rasmussen, KR, Healey, MC, 1992: Experimental Cryptosporidium parvum infection in immunosuppressed adult mice. Infect. Immun. 60: 1648-52.

Santaolalla, R, Abreu, MT, 2012: Innate immunity in the small intestine. Curr. Opin. Gastroenterol. 28:124-9.

Sato, S, Sugiyama, M, Yamamoto, M, Watanabe, Y, Kawai, T, et al, 2003: Toll/IL-1 receptor domain-containing adaptor inducing IFN(TRIF) associates with TNF receptor-associated factor 6 and TANK binding kinase 1, and activates two distinct transcription factors, NF-B and IFN-regulatory factor-3, in the Toll-like receptor signaling. J. Immunol. 171:4304-10.

Striepen, B, 2013: Parasitic infections: time to tackle cryptosporidiosis. Nature 503, 7475:189. Tzipori, S, Griffiths, JK, 1998: Natural history and biology of Cryptosporidium parvum. Adv. Parasitol. 40:5-36.

Ungar, BL, Kao, TC, Burris, JA, Finkelman, FD, 1991: Cryptosporidium infection in an adult mouse model: Independent roles for IFNgamma and CD4 + T lymphocytes in protective immunity. J. Immunol. 147:1014-22.

\section{Explanation of figures}

Fig. 1: C. parvum oocyst shedding in immunosuppressed (DEX) and non-immunosuppressed (controls) mice. Measurements of oocyst shedding from mice at infection different time, values represent mean numbers of oocysts per 10 fields.

Fig. 2a, b, c: Serum levels of acute phase cytokines in infected immunocompetent and immunosuppressed mice compared to control.

Fig. 3a: Sections of small intestine of control mice showed mild expression of TLR4 in villi (red arrow) and crypts (yellow arrow) of intestinal mucosa, as brownish color of cytoplasm lining villi and crypts (Immunohistochemistry, DAB, x200, x400). Fig. 3b: Sections of small intestine of infected non-immunosuppressed mice showed marked expression of TLR4 in villi (red arrow) and crypts (yellow arrow) of intestinal mucosa, as brownish color of cytoplasm lining villi and crypts. Fig. 3c: Sections of small intestine of infected (DEX) immuno- 
suppressed mice showed mild expression of TLR4 in villi (red arrow) and negative or mild expression in crypts of intestinal mucosa (yellow arrow), as brownish color of cytoplasm lining villi and crypts (Immunohistochemistry, DAB, x100, x400).
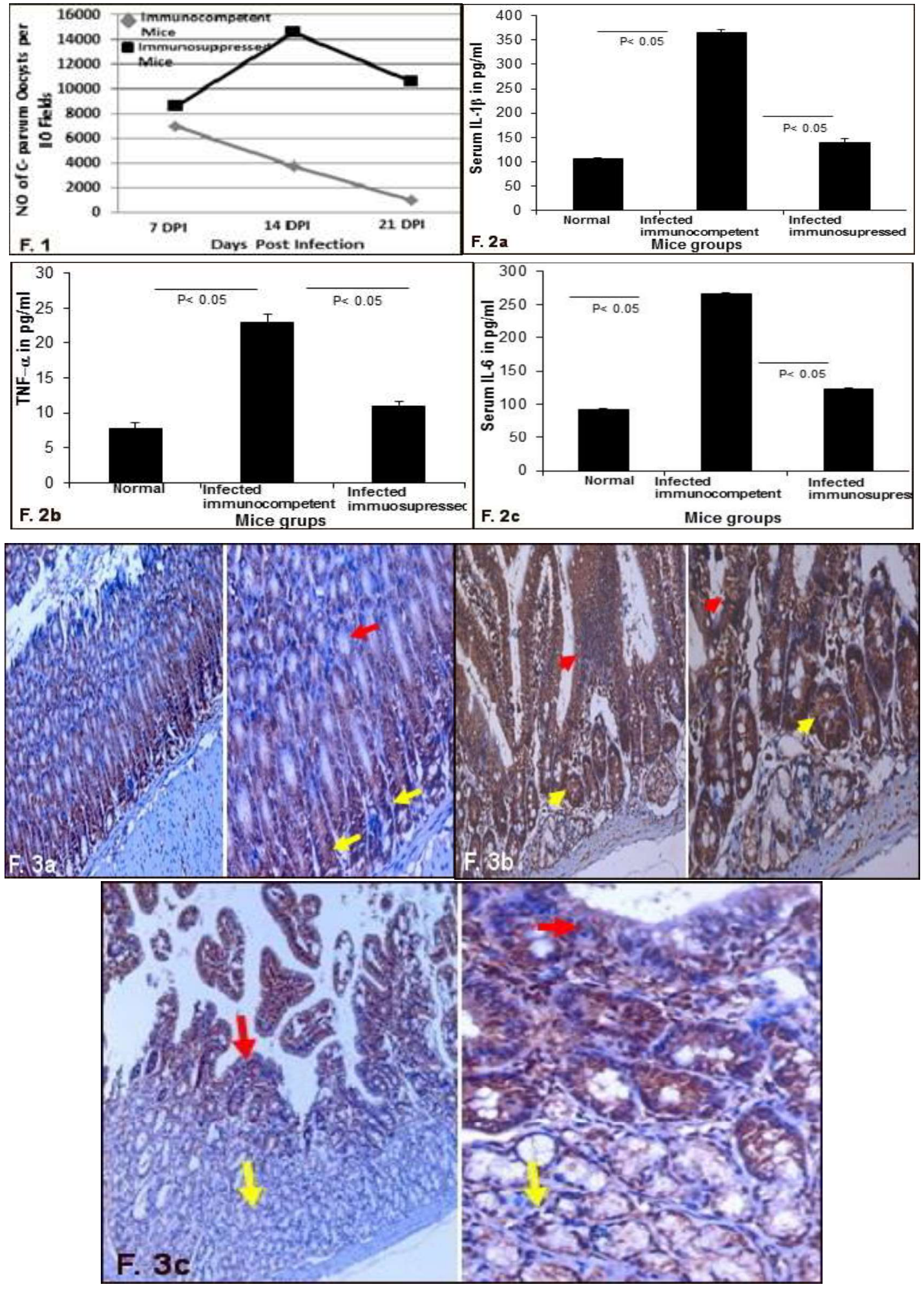